\title{
Éditorial
}

\section{Matériaux utilisés à des fins environnementales - le traitement des pollutions}

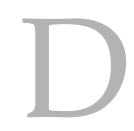

epuis 2002, les congrès « Matériaux » permettent d'identifier au plan national les thématiques fortes, depuis la conception et la fonctionnalisation de matériaux jusqu'aux applications innovantes, en passant par les caractérisations multi-échelles et les modélisations numériques. Après Tours en 2002, puis Dijon en 2006, la troisième édition «Matériaux 2010 », tenue à Nantes, du 18 au 22 octobre 2010, était co-organisée par vingt-trois sociétés savantes rassemblées au sein de la Fédération Française des Matériaux (FFM).

Parmi les nombreuses thématiques liées au domaine des matériaux, celle de leur interaction avec l'environnement a émergé plus récemment. S'il ne fait aucun doute que les matériaux ont largement contribué au progrès et au bien-être des individus, il arrive cependant qu'ils soient parfois pointés du doigt pour leur bilan environnemental. En revanche, on oublie trop souvent qu'un certain nombre de matériaux peuvent être utilisés pour apporter des réponses à des problématiques relatives à la décontamination des matrices environnementales que sont les eaux, l'air, les sédiments aquatiques et les sols.

Le programme était composé de quatorze sessions dont l'une dédiée à la thématique «Matériaux et environnement » (session 12). Cette session a été co-organisée par quatre sociétés savantes : le Groupe Français des Polymères (GFP), l'Association pour les Matériaux Composites (AMAC), le Groupe Français d'Étude des Carbones (GFEC) et le Groupe Français de Mécanique des Matériaux (MECAMAT). Elle s'est déroulée sur quatre jours et a attiré un public nombreux.

Son programme scientifique a permis de présenter 3 conférences invitées, 92 communications orales et 42 posters. Ces communications ont été réparties selon trois thématiques :

- Eco-matériaux, recyclage et matériaux pour le développement durable.

- Matériaux pour le traitement des pollutions.

- ACV - Ecoconception.

Par rapport aux deux précédentes éditions du congrès « Matériaux», la session «Matériaux et environnement » accueillait pour la première fois la thématique "Matériaux pour le traitement des pollutions ", ce qui a permis d'en augmenter largement l'audience. Cette thématique a pour objectif de présenter les matériaux en lien avec le contexte de leur mise en œuvre au sein de procédés destinés à traiter les pollutions présentes dans les eaux naturelles, les effluents, les sédiments aquatiques, les sols et l'air.

Ainsi, dans cette rubrique spéciale du présent numéro de Matériaux \& Techniques, nous publions six articles rédigés sur la base des présentations que nous avons sélectionnées parmi celles de la thématique «Matériaux pour le traitement des pollutions ». Ces articles montrent la diversité des matériaux qui peuvent être utilisés à des fins environnementales, qu'il s'agisse de matériaux naturels comme les apatites, de matériaux synthétiques polymères utilisés dans les géo membranes ou encore de matériaux très sophistiqués comme des nanoparticules de synthèse fonctionnalisées. Ces articles font également ressortir la 
diversité des applications environnementales et procédés industriels de traitement dans lesquels ces matériaux peuvent intervenir pour traiter les eaux, l'air... ou conditionner des déchets pour un stockage de longue durée.

Les éditeurs remercient la Fédération Française des Matériaux pour leur permettre la publication de ces articles basés sur les présentations du congrès.

Vincent Verney

Éditeur en chef

Catherine Morlay

Éditrice invitée 